\title{
Evaluación de la calidad química del humus de lombriz roja californiana (Eisenia foetida) elaborado a partir de cuatro sustratos orgánicos en Arica
}

\author{
Chemical characterization of humus produced by Californian Red Worm \\ (Eisenia foetida) from four organic substrates in Arica
}

\author{
Felipe Salinas-Vásquez, Leslie Sepúlveda-Morales, Germán Sepúlveda-Chavera ${ }^{1 *}$
}

\begin{abstract}
RESUMEN
Se evaluó la calidad química del humus producido por la lombriz roja californiana elaborado a partir de cuatro compost preparados con diferentes desechos orgánicos. Se trabajó con cuatro tratamientos: T0 (suelo 100\%); T1 (compost tomate: pimentón)(1:1 p/p); T2 (restos frescos de tomate-pimentón: suelo)(1:1 p/p); T3 (compost plantas ornamentales Ficus: Hibiscus: Cynodon)(1:1:3 p/p) y T4 (compost olivo: tomate)(1:1 p/p). Cada tratamiento se caracterizó químicamente al iniciar el proceso y después de tres meses. Los resultados se analizaron estadísticamente y se compararon las medias con la prueba de rango múltiple de Tukey (p<0,05). El humus obtenido en T4 (mezcla de herbáceas y leñosas) presentó los mejores indicadores químicos, como reducción del 51,32\% de la $\mathrm{CE}$, reducción del 50\% de la acidez, relación $\mathrm{C} / \mathrm{N}$ de 16, entre otros.
\end{abstract}

Palabras clave: Eisenia foetida, materia orgánica, compost.

\begin{abstract}
The chemical quality of humus produced by the Californian red worm drawn from four different preparations compost organic waste was evaluated. We worked with four treatments: T0 (100\% soil); T1 (compost tomato: capsicum) (1:1 w/w); T2 (fresh tomato, capsicum residues: soil) (1:1 w/w); T3 (compost ornamental plants Ficus : Hibiscus: Cynodon) (1:1:3 w/w) and T4 (olive compost: tomato) $(1: 1 \mathrm{w} / \mathrm{w})$. Each treatment was chemically characterized to start the process and after three months. The results were statistically analyzed and the means were compared with the multiple range test of Tukey $(p<0.05)$. The humus obtained in T4 (mixture of herbaceous and woody) presented the best chemical indicators such as reduction of 51.32\% EC, 50\% reduction in acidity, $C / N$ ratio of 16, among others.
\end{abstract}

Key word: Eisenia foetida, organic matter, compost.

\section{Introducción}

Los procesos pedogénicos y la situación biogeográfica del valle de Azapa en la Región de Arica y Parinacota, Chile, determinan que los suelos presenten niveles mínimos de materia orgánica, mientras que la $\mathrm{CE}$ es mayor a $2 \mathrm{dS} / \mathrm{m}$, exhibiendo algún grado de salinidad a causa del agua de riego (Riquelme-Garcés et al., 2013; Mazuela, 2013). Aun así la agricultura ha logrado establecerse y ser un negocio rentable, basado en la producción de hortalizas fuera de temporada (Riquelme-Garcés et al., 2013). El equilibrio edáfico es inestable y existen varias prácticas que deben mejorarse. Tales prácticas contribuyen a empobrecer el recurso edáfico, disminuyendo la macro y microfauna, alterando negativamente el equilibrio microbiológico, acelerando la salinización y favoreciendo la degradación en el tiempo.

La integración de prácticas adecuadas de manejo del suelo a largo plazo adquieren importancia en su conservación. El suelo debe ser considerado un ente vivo y, en la medida que aumenta la presencia de microorganismos, las características físicas, químicas y biológicas de este serán más adecuadas para el desarrollo de los cultivos (Moreno, 1996).

Es necesario incorporar criterios de manejo agrícola sustentable, integrando sistemas y prácticas orgánicas y de reciclaje, que tiendan a satisfacer indefinidamente las necesidades del consumo de

\footnotetext{
1 Fac. Cs. Agronómicas, U. Tarapacá, Avda. General Velásquez 1775, Arica, Chile.

* Autor para correspondencia: gsepulve@uta.cl
} 
la población, sin degradar los recursos naturales que la hacen posible (Altieri y Nicholls, 2000). Los desechos orgánicos generados en la agricultura representan un potencial enorme en el manejo ecológico y nutricional del suelo (Julca-Otiniano et al., 2006).

La incorporación de materia orgánica como acondicionador es una buena gestión en el manejo de suelos, repone los nutrientes extraídos por los cultivos, mientras mejora las propiedades físicas, químicas y biológicas a largo plazo (Álvarez de Brito et al., 1995). En la gama de alternativas para la adición de materia orgánica al suelo destaca la incorporación de humus de lombriz o vermicompuesto, debido a que posee gran estabilidad, elevado contenido en fibra bacteriana $\mathrm{y}$ alto contenido de nutrientes asimilables para las plantas (Manaf et al., 2009).

La lombricultura es una técnica orgánica, en la que por medio del manejo de procesos naturales en el suelo permite favorecer su dinámica y como consecuencia, obtener un impacto positivo en el ámbito agrícola (Hatti et al., 2010), social y económico (Gheisari et al., 2010). La aplicación de humus mejora la germinación y crecimiento de diferentes especies (Subler et al., 1998; Atiyeha et al., 2000) por la cantidad de nutrientes presentes en el vermicompuesto y que no se encuentran totalmente en los fertilizantes químicos, como nitrógeno, fósforo, potasio soluble, así como calcio y magnesio (Orozco et al., 1996).

El objetivo de este trabajo fue evaluar las principales características químicas del humus producido por la lombriz roja californiana (Eisenia foetida) elaborado a partir de diferentes desechos orgánicos en el valle de Azapa.

\section{Materiales y Métodos}

\section{Preparación del sustrato (compost)}

Se evaluó cuatro sustratos preparados con diferentes mezclas de material vegetal y suelo proveniente del valle de Azapa. Cada compost se preparó de acuerdo con la norma chilena ( $\mathrm{NCh}$ 2880), tres meses antes de exponerlo a la acción de E. foetida. Los tratamientos fueron: suelo agrícola del valle de Azapa $100 \%$ (T0); compost elaborado con rastrojos de tomate y pimentón (1:1 $\mathrm{p} / \mathrm{p})(\mathrm{T} 1)$, mezcla de suelo y rastrojos de tomate $(1: 1 \mathrm{p} / \mathrm{p})(\mathrm{T} 2)$, compost elaborado con rastrojos ornamentales (Ficus sp., Hibiscus sp., Cynodon sp. $(1: 1: 3 \mathrm{p} / \mathrm{p})(\mathrm{T} 3)$ y compost elaborado con rastrojos de olivo y tomate $(1: 1 \mathrm{p} / \mathrm{p})(\mathrm{T} 4)$. Las mezclas de material se picaron y homogenizaron revolviendo vigorosamente y formando camas de compostaje de $20 \times 2 \times 1 \mathrm{~m}$ de longitud $\mathrm{x}$ ancho y $\mathrm{x}$ alto. Estas camas se regaron dos veces a la semana durante tres meses, manteniendo la temperatura menor a $50{ }^{\circ} \mathrm{C}$. Cada cama de compostaje se volteó cada 20 días con pala hasta el término del proceso.

\section{Preparación de humus}

Una vez estabilizado el compost se establecieron los tratamientos. Para ello, en bandejas de madera de $40 \times 30 \times 5 \mathrm{~cm}$ (largo x ancho x profundidad), con un fondo doble de malla plástica para favorecer el drenaje, se colocaron $4 \mathrm{~kg}$ de compost y se inocularon con 100 individuos clitelados de E. foetida. Las bandejas se dispusieron bajo malla sombra $80 \%$ para evitar el exceso de luminosidad y el ataque de depredadores (aves). Una vez inoculadas las bandejas se regaron diariamente para mantener la humedad del sustrato a $80 \%$. Después de 90 días se rescató el humus para su evaluación y caracterización.

\section{Determinaciones de laboratorio}

El análisis químico de los sustratos antes de ser inoculados con lombrices y 90 días después, una vez humificados, se hicieron en el Laboratorio de Análisis Químico de Suelos y Aguas de la Universidad de Tarapacá. De cada unidad experimental se tomó $500 \mathrm{~g}$ de muestra compuesta. Después de secarla al aire se tamizó con una malla de $2 \mathrm{~mm}$ de diámetro. Se midió la acidez $(\mathrm{pH})$ usando el método potenciométrico con una relación suelo:agua 1:2,5 (método INIA 3-3.1). La salinidad se evaluó mediante el método conductimétrico (método INIA 9-9.1). Para determinar los niveles de materia orgánica se trabajó con el método INIA 7.7.1, donde se oxida la materia orgánica con $\mathrm{K}_{2} \mathrm{Cr}_{2} \mathrm{O}_{7}$ en medio considerablemente ácido. Con posterioridad se mide la absorbancia de la solución resultante en espectrofotómetro de absorción molecular (pfp 7, Jenway, Reino Unido) a una longitud de onda de $600 \mathrm{~nm}$ (Sadzawka et al., 2006).

El nitrato disponible en cada sustrato se determinó por medio de extracción con $\mathrm{Ag}_{2} \mathrm{SO}_{4}$ $(0,01 \%)$ y determinación colorimétrica con fenoldisulfónico a $420 \mathrm{~nm}$ (Jackson, 1978). Para 
determinar nitrógeno orgánico se trabajó con el método de Kjendahl, que consiste en una digestión de la muestra seca y molida (tamizada por malla de $2 \mathrm{~mm}$ ) con $\mathrm{H}_{2} \mathrm{SO}_{4}$ y catalizador mercurio metálico y destilación de la sal de amonio formada como amoniaco sobre solución de ácido bórico, para titular con $\mathrm{HCl}$ 0,100N (método INIA 5-5.1).

\section{Diseño experimental y análisis estadístico}

El ensayo se estableció en condiciones de invernadero, con un diseño experimental de bloques completos al azar con cuatro tratamientos, un testigo absoluto y tres repeticiones. Los datos se sometieron a pruebas de normalidad (Test de Shapiro Wilk), Test de Levene para determinar la homogeneidad de varianza. Los datos se sometieron al análisis de Kruscal-Wallis y a la prueba de comparación de medias de Tuckey $(\mathrm{p}=0,05)$. Se trabajó con el paquete estadístico Minitab (Minitab Inc, software shop, 1972).

\section{Resultados y Discusión}

Los parámetros químicos iniciales de los sustratos evaluados fueron adecuados para el desarrollo y reproducción de la lombriz roja californiana. La acidez, evaluada como $\mathrm{pH}$, de todos los sustratos fluctuó entre 8,67 y 7,86, correspondiente a los tratamientos T2 y $\mathrm{T} 3$, respectivamente. La conductividad eléctrica se redujo en todos los tratamientos. El tratamiento T1 presentó una reducción del 73,06\%, mientras que los restantes tratamientos redujeron la CE entre 18 y $51 \%$ (Tabla 1). El compost del T1 presentó un pH de 8,5, el que sobrepasó los límites indicados en la NCH 2880 para ser clasificado como compost clase A o B. El tratamiento T3 se pudo clasificar como compost clase $\mathrm{A}$, y el tratamiento $\mathrm{T} 4$ como compost clase B, debido a su alta $\mathrm{CE}\left(3,94 \mathrm{mS} / \mathrm{cm}^{2}\right)$.

La materia orgánica aumentó en todos los tratamientos excepto en T2. En el tratamiento T0, aun cuando no se aportó material vegetal, el incremento fue de $114 \%$, debido a la acción de $E$. foetida y al incremento en la biomasa microbiana (Aria y Domínguez, 2010). En los otros tratamientos el incremento fue variable, debido a que el material con el que se preparó el sustrato posee características diferentes. El T2 incluyó materia orgánica no estabilizada, registrando una pérdida de $14 \%$, debido a que esta materia orgánica fresca se descompone con rapidez en formas inorgánicas simples, mineralizándose (Yague, 1999).

En T0, T1, T3 y T4 el incremento en la relación $\mathrm{C} / \mathrm{N}$ se relaciona con el leve descenso del contenido de $\mathrm{N}$ total, ya que el nitrógeno orgánico debe convertirse

Tabla 1. Parámetros químicos de los sustratos. T0: Suelo agrícola; T1: Compost tomate: pimentón (1:1);

T2: Mezcla de suelo: tomate (1:1); T3: Compost ornamentales; T4: Compost olivo: tomate (1:1), antes de ser inoculados (Columna I) y después de 90 días de humificación (Columna F). Tercera columna: Tasa de cambio $(\Delta \%)$. Cada valor representa el promedio de 4 repeticiones.

\begin{tabular}{|c|c|c|c|c|c|c|c|c|}
\hline & \multirow[b]{2}{*}{ Tratamiento } & \multicolumn{7}{|c|}{ Parámetros } \\
\hline & & $\begin{array}{c}\mathrm{Ce} \\
(\mathrm{dS} / \mathrm{m})\end{array}$ & $\begin{array}{c}\text { Acidez } \\
(\mathrm{pH})\end{array}$ & $\begin{array}{c}\text { Mat. orgánica } \\
(\%)\end{array}$ & $\begin{array}{c}\text { Relación } \\
\text { C/N }\end{array}$ & $\begin{array}{c}\text { Nitrógeno } \\
(\%)\end{array}$ & $\begin{array}{c}\text { Fósforo } \\
(\%)\end{array}$ & $\begin{array}{c}\text { Potasio } \\
(\%)\end{array}$ \\
\hline \multirow{3}{*}{ T0 } & Inicial & 2,97 & 8,62 & 3,21 & 3,5 & 0,99 & 0,085 & 277,5 \\
\hline & Final & $0,8 \mathrm{~d}$ & $8,6 \mathrm{a}$ & $6,9 \mathrm{e}$ & $4,51 \mathrm{e}$ & $0,98 \mathrm{~d}$ & $0,07 \mathrm{c}$ & $519,68 \mathrm{~b}$ \\
\hline & $\Delta \%$ & $-73,06$ & $-0,23$ & 114,95 & 28,86 & $-1,01$ & $-17,65$ & 87,27 \\
\hline \multirow{3}{*}{$\mathrm{T} 1$} & Inicial & 2,6 & 8,5 & 35 & 16 & 1,3 & 0,36 & 3.611 \\
\hline & Final & $2,13 \mathrm{a}$ & $8,5 \mathrm{a}$ & $37,9 \mathrm{~b}$ & $17,46 \mathrm{a}$ & $1,25 \mathrm{c}$ & $0,13 \mathrm{a}$ & $470,4 \mathrm{c}$ \\
\hline & $\Delta \%$ & $-18,08$ & 0,00 & 8,29 & 9,13 & $-3,85$ & $-63,89$ & $-86,97$ \\
\hline \multirow{3}{*}{$\mathrm{T} 2$} & Inicial & 1,72 & 8,67 & 15 & 9,7 & 0,9 & 0,198 & $5.055,55$ \\
\hline & Final & $0,88 \mathrm{e}$ & $8,6 \mathrm{a}$ & $12,9 \mathrm{~d}$ & $8,02 \mathrm{~d}$ & $0,94 \mathrm{e}$ & $0,1 \mathrm{~b}$ & $522 \mathrm{a}$ \\
\hline & $\Delta \%$ & $-48,84$ & $-0,81$ & $-14,00$ & $-17,32$ & 4,44 & $-49,49$ & $-89,67$ \\
\hline \multirow{3}{*}{$\mathrm{T} 3$} & Inicial & 2,32 & 7,86 & 31,2 & 9,76 & 1,86 & 0,2 & 3.230 \\
\hline & Final & $1,38 \mathrm{c}$ & $7,86 \mathrm{~b}$ & $31,6 \mathrm{c}$ & $14,4 \mathrm{c}$ & $1,32 \mathrm{~b}$ & $0,12 \mathrm{a}$ & $388,8 \mathrm{~d}$ \\
\hline & $\Delta \%$ & $-40,52$ & 0,00 & 1,28 & 47,54 & $-29,03$ & $-40,00$ & $-87,96$ \\
\hline \multirow{3}{*}{$\mathrm{T} 4$} & Inicial & 3,97 & 7,94 & 36 & 14 & 1,65 & 0,4 & 3.500 \\
\hline & Final & $1,93 \mathrm{~b}$ & $7,9 \mathrm{~b}$ & $44,3 \mathrm{a}$ & $16,05 \mathrm{~b}$ & $1,6 \mathrm{a}$ & $0,13 \mathrm{a}$ & $380 \mathrm{e}$ \\
\hline & $\Delta \%$ & $-51,39$ & $-0,50$ & 23,06 & 14,64 & $-3,03$ & $-67,50$ & $-89,14$ \\
\hline
\end{tabular}


a la forma inorgánica, cuyas formas varían según el origen del material (Castillo et al., 2000). De acuerdo con Gómez-Brandon y colaboradores (2010), las lombrices favorecen la nitrificación, causando la rápida conversión del nitrógeno amoniacal en nitratos, aumentando la mineralización de nitrógeno. Durante los procesos de transformación de nitrógeno en el suelo la materia orgánica no estabilizada al mineralizarse genera excedentes de $\mathrm{N}$, proceso contrario al que fue registrado en los otros tratamientos, donde se observó pérdida neta de $\mathrm{N}$, lo que es ratificado por Yague (1999).

Los niveles de fósforo registraron pérdidas que van de 17,6 al 63,89\%, las que se pueden explicar por lixiviación y arrastre. El parámetro más estable, y por lo tanto independiente del origen del sustrato, es el potasio. Todos los tratamientos presentaron una reducción del $\mathrm{K}$ disponible de $88,4 \%$ en promedio, excepto el tratamiento T0, en el que se incrementó 87,3\%.

Los sustratos evaluados fueron óptimos para el crecimiento y desarrollo de E. foetida, oligoqueto que al actuar sobre sustratos con restricción de uso agrícola los transforma dejándolos aptos para su aplicación en cualquier cultivo. La combinación de diferentes materiales vegetales usados como sustrato, principalmente combinaciones de herbáceas y leñosas, generan un sustrato de alto valor biológico estabilizado por la acción de microorganismos y anélidos oligoquetos.

\section{Agradecimientos}

Los autores agradecen al proyecto Mayor UTA 9711-13 y a la señora Patricia Pacheco por el apoyo en los análisis químicos.

\section{Literatura Citada}

Aira, M.; Domínguez, J.

2010. Las lombrices de tierra y los microorganismos: desentrañando la caja negra del vermicompostaje. Acta Zoológica Mexicana, (n.s.) Número Especial 2: 385-395.

Altieri, M.; Nicholls, C.I.

2000. Teoría y práctica para una agricultura sustentable. Serie Textos Básicos para la Formación Ambiental. PNUMA. Red de Formación Ambiental para América Latina y el Caribe. México, $235 \mathrm{pp}$.

Álvarez de Brito, Ma.; Gagne, S.; Antoun, H.

1995. Effect of compost on Rhizosphere Microflora of the tomato and on the Incidence of Plant Growth Promoting Rhizobacteria. Appl. Environ. Microbiol, 61 (1): 194-199.

Atiyeha, R.M.; Sublera, S.; Edwardsa, C.A.; Bachmanb, G.;

Metzgerb, J.D.; Shustera, W.

2000. Effects of vermicomposts and composts on plant growth in horticultural container media and soil. Pedobiologia, 44 (5): 579-590.

Castillo, A.; Quarín, S.; Iglesias, M.

2000. Caracterización química y física de compost de lombrices elaborado a partir de residuos orgánicos puros y combinados. Agric. Téc., 60 (1): 74-79.

Gheisari, S.; Danesh, S.; Mousavi, S.M.

2010. Growth and Reproduction of Eisenia foetida in Vermicomposting of Organic Fraction of Municipal Solid Wastes. Asian Journal of Chemistry, 22 (2): 1266-1274.

Gómez-Brandón, M.; Lazcano, C.; Lores, M.; Domínguez, J. 2010. Papel de las lombrices de tierra en la degradación del bagazo de uva: efectos sobre las características químicas y la microflora en las primeras etapas del proceso. Acta Zoológica Mexicana, Número especial 2: 397-408.

Hatti, S.S.; Londonkar, R.L.; Patil, S.B.; Gangawane, A.K.; Patil, C.S.

2010. Effect of Eisenia foetida vermiwash on the growth of plants. Journal of Crop Science, 1 (1): 6-10.
Julca-Otiniano, A.; Meneses-Florian, L.; Blas-Sevillano, R. 2006. La materia orgánica, importancia y experiencia de su uso en la agricultura. Idesia (Chile) 24 (1): 49-61.

Labrador, $\mathrm{M}$.

2002. La materia orgánica en los agrosistemas. Segunda edición. Madrid-España, Ediciones Mundi-Prensa 293 pp.

Manaf, L.A.; Jusoh, M.L.C.; Yusoff, M.K.; Ismail, T.H.T.; Harun,

R.; Juahir, H.; Jusoff, K.

2009. Influences of Bedding Material in Vermicomposting Process. International Journal of Biology, 1 (1): 81-91.

Mazuela, A., P.

2013. Agricultura en zonas áridas y semiáridas. Idesia, 31 (2): 3-4.

Mejías, P.

2005. Manual Lombricultura. Ed. Agroflor. Chile. 54 pp.

Moreno, J.; Moral, R.

2008. Compostaje. Madrid, España. Ediciones Mundi-Prensa. $570 \mathrm{pp}$.

Orozco, F.H.; Cegarra, J.; Trujillo, L.M.; Roig, A.

1996. Vermicomposting of coffee pulp using earthworm Eisenia foetida: Effects on $\mathrm{C}$ and $\mathrm{N}$ contents and the availability of nutrients. Biology and Fertility of Soils, 22: 162-166.

Pineda, J.

2006. Lombricultura. Primera edición. Tegucigalpa-Honduras (Ed. Instituto Hondureño del Café). 7-11 pp.

Riquelme-Garcés, A.; González-Vallejos, F.; Contreras-Luque, P.; Mazuela, A., P.

2013. Manejo del cultivo de hortalizas y su efecto en la sustentabilidad de un valle costero del desierto de Atacama, Chile. Idesia, 31 (3): 113-117.

Moreno, J.L.

1996. La materia orgánica en los agrosistemas: Ministério de Pesca, Agricultura y Alimentación. Primera coedición. Madrid, España. 174 pp. 
Sadzawka R., A.; Carrasco R., M.A.; Grez Z., R.; Mora G., M.L.; Flores P., H.; Neaman, A.

2006. Métodos de análisis de suelos recomendados para los suelos de Chile. Revisión 2006. Instituto de Investigaciones Agropecuarias, Serie Actas INIA No 34 , Santiago, Chile, $164 \mathrm{pp}$.
Subler, S.; Edwards, C.A.; Metzger, J.

1998. Comparing vermicompost and compost. Biocycle. 39: 63-66.

Yague, J.

1999. El suelo y los fertilizantes. Madrid-España, Ediciones Mundi-Prensa. 104 pp. 
